\title{
T315I Mutation in Philadelphia Chromosome-positive Acute Lymphoblastic Leukemia: Role for Detection of Mutations
}

Sir,

We report a patient with Philadelphia-positive acute lymphoblastic leukemia (ALL) $\left(\mathrm{Ph}^{+} \mathrm{ALL}\right)$ who failed to respond to first-line tyrosine kinase inhibitors (TKIs) and was subsequently detected to have T315I mutation in the BCR-ABL kinase domain. This has significant therapeutic implications in the form of need for early initiation of second-line TKIs and justifies early allogeneic stem cell transplant; even if haploidentical or matched unrelated donors are only available.

A 25-year-old male presented with fatigue, myalgia, and lymphadenopathy. Initial investigations (normal range) showed hemoglobin, $6 \mathrm{~g} \%$ (12-15); total leukocyte count, $65,000 / \mathrm{cu} \mathrm{mm}(4000-10,000)$; and platelet count, $58,000 / \mathrm{cu} \mathrm{mm} \quad(150,000-450,000)$. Differential count showed $80 \%$ lymphoblasts in the peripheral blood. Flow cytometry of bone marrow aspirate confirmed the diagnosis of precursor B-cell ALL. Cerebrospinal fluid examination was normal. Cytogenetic examination of bone marrow revealed $46 \mathrm{XY}, \mathrm{t}(9 ; 22)(\mathrm{q} 34 ; \mathrm{q} 11.2)$ in all 20 metaphases. Qualitative reverse transcriptase polymerase chain reaction (RT-PCR) revealed a minor translocation in BCR-ABL involving exons e2' (bcr) and e2 (abl) resulting in a $190 \mathrm{kD}$ fusion protein.

He received intensive chemotherapy as per the Berlin Frankfurt Munster (BFM)-95 protocol along with imatinib mesylate $600 \mathrm{mg}$ daily. His bone marrow was in morphological remission after Phase I and he continued with further treatment which included Phase II of induction, consolidation, and reinduction. The $B C R-A B L$ transcript was detectable in the peripheral blood by quantitative RT-PCR at the end of consolidation and after reinduction. Allogeneic stem cell transplantation was not performed as suitable donors were not available.

He commenced maintenance therapy with 6-mercapto-purine and methotrexate. In view of the persistent $B C R-A B L$-positive status in the peripheral blood at the end of reinduction, imatinib was stopped and dasatinib was commenced at $140 \mathrm{mg}$ /day along with maintenance chemotherapy. Three months later, he had a relapse of disease in the bone marrow. Analysis of peripheral blood by nested RT-PCR and Sanger's sequencing identified a T315I mutation. He was treated subsequently with BFM- 86 protocol and a trial of axitinib for 15 days. Unfortunately, his disease was refractory and continued to progress. Upon treatment with fludarabine, idarubicin and high-dose cytarabine (FLAG-IDA regimen), his bone marrow was in complete remission. Following this, he underwent a haploidentical allogeneic stem cell transplantation, the donor being the mother. He expired on the $15^{\text {th }}$ day due to transplant-related complications.
Although TKIs have improved the outcomes of $\mathrm{Ph}^{+} \mathrm{ALL}$, the majority of patients with $\mathrm{Ph}^{+}$ALL ultimately relapse despite using combinations of chemotherapy, first- or second-generation TKIs. Currently, the only curative option is allogeneic peripheral blood stem cell transplantation. ${ }^{[1]}$ Unlike chronic myeloid leukemia (CML), where significant proportions of patients who fail first-line therapy with imatinib remain sensitive to second-line TKIs such as dasatinib or nilotinib, this is not the case with $\mathrm{Ph}^{+}$ALL. ${ }^{[2,3]}$ Dasatinib resistance occurs in $94 \%$ of patients who started this drug after imatinib failure and $80 \%$ of these have $B C R-A B L$ kinase domain mutations. ${ }^{[2]}$ These mutations may be present at baseline or may evolve during therapy. ${ }^{[2]}$ The higher rates of resistant mutations compared to those seen in patients of CML (20\%-40\%) may be explained by the differences in biology between the two diseases. ${ }^{[2,3]}$ This patient failed both imatinib and dasatinib because of the T315I mutation which was only identified later.

T315I mutation in the P-loop of the kinase domain of the fusion Bcr-Abl transcript is the most common mutation (37\%) noted in $\mathrm{Ph}^{+}$ALL patients when their disease is resistant to imatinib. ${ }^{[2]}$ The prevalence of T315I mutation is even higher $(65 \%-79 \%)$ among patients who are on second-line dasatinib after imatinib failure. ${ }^{[2]}$ Limited therapeutic options exist for patients with T315I-mutated leukemia. Ponatinib is approved for this indication in CML, but we were unable to procure this drug for this patient. ${ }^{[4]}$ Recent reports suggest that axitinib might have a role; however, the disease did not respond in this case. ${ }^{[5]}$

Evaluation for mutations in the kinase domain of BCR-ABL transcript is done less frequently in $\mathrm{Ph}^{+} \mathrm{ALL}$ when compared to CML where there exist strict guidelines mandating testing at early signs of molecular failure. This case illustrates the need to consider for evaluation of mutations in all patients with $\mathrm{Ph}^{+}$ALL who develop resistance to therapy. If drug-sensitive mutations are identified, alternative TKIs can be considered. Even though HLA-matched allogeneic stem cell transplants are currently standard of care in $\mathrm{Ph}^{+} \mathrm{ALL}$, even alternate donor or haploidentical transplants may be justified in situations where resistant mutations are identified and are likely to work better if instituted before the onset of morphological relapse.

Financial support and sponsorship

Nil.

Conflicts of interest

There are no conflicts of interest. 


\section{Anjana Joel, Prasanth Ganesan, Krishnarathnam Kannan, Venkatraman Radhakrishnan, Trivadi S Ganesan, Tenali Gnana Sagar}

Department of Medical Oncology, Cancer Institute (WIA), Chennai, Tamil Nadu, India

Address for correspondence: Dr. Prasanth Ganesan, Department of Medical Oncology, Cancer Institute (WIA), Chennai, Tamil Nadu, India. E-mail:pg1980@gmail.com

\section{References}

1. Liu-Dumlao T, Kantarjian H, Thomas DA, O'Brien $\mathrm{S}$, Ravandi F. Philadelphia-positive acute lymphoblastic leukemia: Current treatment options. Curr Oncol Rep 2012;14:387-94.

2. Soverini S, De Benedittis C, Papayannidis C, Paolini S, Venturi C, Iacobucci I, et al. Drug resistance and BCR-ABL kinase domain mutations in Philadelphia chromosome-positive acute lymphoblastic leukemia from the imatinib to the second-generation tyrosine kinase inhibitor era: The main changes are in the type of mutations, but not in the frequency of mutation involvement. Cancer 2014;120:1002-9.

3. Yeung DT, Moulton DJ, Heatley SL, Nievergall E, Dang P, Braley J, et al. Relapse of BCR-ABL1-like ALL mediated by the ABL1 kinase domain mutation T315I following initial response to dasatinib treatment. Leukemia 2015;29:230-2.
4. Sanford DS, Kantarjian H, O'Brien S, Jabbour E, Cortes J, Ravandi F. The role of ponatinib in Philadelphia chromosome-positive acute lymphoblastic leukemia. Expert Rev Anticancer Ther 2015;15:365-73.

5. Pemovska T, Johnson E, Kontro M, Repasky GA, Chen J, Wells $\mathrm{P}$, et al. Axitinib effectively inhibits BCR-ABL1(T315I) with a distinct binding conformation. Nature 2015;519:102-5.

This is an open access journal, and articles are distributed under the terms of the Creative Commons Attribution-Non Commercial-ShareAlike 4.0 License, which allows others to remix, tweak, and build upon the work non-commercially, as long as appropriate credit is given and the new creations are licensed under the identical terms.

\begin{tabular}{|l|l|}
\hline \multicolumn{2}{|c|}{ Access this article online } \\
\hline Quick Response Code: & Website: \\
& www.ijmpo.org \\
\cline { 2 - 2 } & DOI: \\
\hline
\end{tabular}

How to cite this article: Joel A, Ganesan P, Kannan K, Radhakrishnan V, Ganesan TS, Sagar TG. T315I mutation in Philadelphia chromosome-positive acute lymphoblastic leukemia: Role for detection of mutations. Indian J Med Paediatr Oncol 2018;39:417-8.

C 2018 Indian Journal of Medical and Paediatric Oncology | Published by Wolters Kluwer - Medknow 